\title{
Insulin resistance and the disruption of Glut4 trafficking in skeletal muscle
}

\author{
Mike Mueckler \\ Department of Cell Biology and Physiology, Washington University School of Medicine, Box 8228, St. Louis, \\ Missouri 63110, USA. Phone: (314) 362-4160; Fax: (314) 362-7463; E-mail: mike@cellbio.wustl.edu.
}

The facilitative transport of glucose across animal cell membranes is mediated by members of the Glut protein family (1). These 50 - to $60-\mathrm{kDa}$ glycoproteins are ubiquitously expressed in mammalian tissues and are responsible for the uptake of sugar from the blood into cells, supplying cellular glucose for ATP production and for a wide variety of anabolic reactions. Additionally, glucose transport in certain tissues plays a critical role in whole-body glucose homeostasis. During fasting or starvation, the liver and kidney maintain euglycemia by becoming net producers of blood glucose, initially via the breakdown of glycogen stores and later through the catabolism of amino acids. Conversely, when the insulin level rises during the postprandial period in response to ingested carbohydrates, fat and muscle tissue become the major sites of glucose metabolism and storage.

The appropriate output or uptake of glucose from these tissues via the Glut proteins is critical to health and survival. For example, a disruption in the normal process by which insulin stimulates glucose transport into skeletal muscle is the cause of the peripheral insulin resistance associated with non-insulin-dependent diabetes mellitus (NIDDM) (2). Insulin resistance is the earliest detectable abnormality observed during the natural history of NIDDM and is believed to be a major triggering event in the development of the disease (3). Thus, a detailed understanding of how insulin regulates glucose transport in muscle is essential to unraveling the molecular basis of NIDDM. In this issue, Yang et al. (4) identify syntaxin 4 as one of the key molecular components in insulin-stimulated glucose transport in skeletal muscle. They also provide evidence supporting a novel mechanism for the pathogenesis of insulin resistance the downregulation of a factor involved in glucose transporter subcellular trafficking.

\section{Insulin-responsive glucose transport in the adipocyte} Insulin-responsive glucose transport has been exhaustively studied in the adipocyte since 1980 , when evidence came to light that insulin stimulates the recruitment or translocation of glucose transporters from intracellular membrane compartments to the plasma membrane, increasing the number of functional cell surface glucose transporters and thus the rate of sugar uptake $(5,6)$. Overwhelming experimental evidence for this mechanism has accumulated during the past two decades, driven largely by the identification of the insulin-responsive glucose transporter isoform Glut4 (7-9). Adipocytes express at least two glucose transporter isoforms, Glut1 and Glut4 (10), but Glut 4 is by far the more abundant isoform and appears to be responsible for most or all of the acute increase in glucose transport stimulated by insulin (11). Following a poorly defined pathway, Glut4 recycles through endosomes and the adipocyte plasma membrane in the unstimulated steady state. Because the endocytic rate exceeds the exocytic rate $(12,13)$, the transporter is largely excluded from the cell surface under basal conditions. Glut4 is thus enriched in intracellular membrane compartments, including what appears to be a unique insulinsensitive compartment that morphologically resembles the trans-Golgi reticulum $(14,15)$. Acting through a poorly understood signaling pathway, insulin brings about a change in the endocytic and exocytic rate constants, such that about $50 \%$ of intracellular Glut 4 redistributes to the plasma membrane, greatly increasing the rate of glucose transport $(12,13)$.

Some elements of the molecular machinery involved in regulated Glut 4 trafficking in the adipocyte have now been identified. Insulin-stimulated redistribution of Glut 4 from intracellular compartments to the plasma mem- brane is believed to occur via a vesiclemediated fusion process analogous to the postulated model for neurotransmitter release at the presynaptic terminus (16). Glut4-containing transport vesicles are postulated to bud from an insulin-sensitive intracellular membrane compartment and to fuse with the plasma membrane. This fusion is mediated by vesicle-associated SNARE proteins (v-SNAREs) present in transport vesicles, which interact specifically with target-membrane SNARE proteins ( $t$-SNAREs) in the plasma membrane. In adipocytes, the $\mathrm{v}$-SNARE and $\mathrm{t}$ SNARE that appear to dictate Glut4 vesicle fusion with the plasma membrane are VAMP2 $(17,18)$ and syntaxin 4/SNAP23 $(18,19)$, respectively. Additionally, the accessory proteins, Munc18c $(20,21)$ and Synip (22), may be involved in regulating the interaction between VAMP2 and syntaxin 4/SNAP23 in an insulin-responsive fashion, although their precise roles in regulated vesicle fusion have not yet been elucidated. Several different models have been postulated for the vesicular route(s) followed by Glut 4 as it traverses the cell in response to changing insulin levels. Kinetic analysis suggests that at least three distinct membranous compartments are involved (23), but the precise Glut 4 trafficking pathways remain ill-defined.

\section{Insulin-responsive glucose transport in the muscle fiber}

Much less is known about the Glut4 trafficking pathway and its associated molecular machinery in the muscle fiber in comparison with the adipocyte. Progress in this area has been hampered by difficulties in performing traditional cell biological procedures on skeletal muscle tissue, combined with a lack of muscle cell lines that closely reflect the anatomy and physiology of striated muscle fibers and the difficulty of expressing ectopic proteins in intact skeletal muscle. Like adipocytes, muscle 
fibers express both Glut1 and Glut4, with Glut4 being vastly more abundant (24). As in the adipocyte, Glut4 appears to be responsible for most or all of the insulin-stimulated glucose transport that occurs in muscle fibers (25). In contrast to adipocytes, skeletal muscle fibers contain not one but two distinct plasma membrane domains: the sarcolemma (or plasma membrane proper) and the transverse tubules (t-tubules), special invaginations of the plasma membrane that extend deep into the fiber. T-tubules and the sarcolemma have distinct protein compositions and represent two distinct subcellular targeting domains (26). In skeletal muscle, Glut 1 is targeted constitutively to the sarcolemma and is largely excluded from t-tubules, and, unlike in adipocytes, Glut1 is not responsive to insulin (27). In contrast, Glut4 is enriched in membrane structures in the triad region of muscle fibers, where the t-tubules, terminal cisternae, and sarcoplasmic reticulum meet, as well as in the sarcolemma, subsarcolemmal vesicles, and perinuclear Golgi-like membrane structures $(27,28)$. Insulin stimulates an increase in the density of Glut4 both in the sarcolemma and in ttubules, but the bulk of insulin-stimulated transport appears to occur across t-tubules (27). This latter observation is consistent with quantitative morphological analyses indicating that more than $90 \%$ of Glut 4 in the insulin-stimulated state is present in t-tubules (27), which generally present a much greater total surface area to the extracellular milieu in muscle fibers than does the sarcolemma. This finding apparently explains how glucose can permeate the dense myofibrillar network and diffuse deep into the muscle fiber, where it is required for ATP production and biosynthetic reactions.

\section{Insulin-responsive glucose transport in adipocytes and muscle fibers}

Yang et al. in this issue of the JCI (4) generated and characterized genetically altered mice that lack a functional syntaxin 4 gene (Syn4). The authors observe that homozygous disruption of Syn4 causes early embryonic lethality, but that mice lacking only one functional Syn4 allele develop normally despite the fact that their steady-state levels of syntaxin 4 protein are decreased in many tissues. However, under euglycemic/hyperinsu- linemic clamp conditions, the heterozygous knockout mice exhibit impaired glucose tolerance accompanied by an approximately $50 \%$ reduction in insulinstimulated glucose uptake into muscle and a proportional decrease in wholebody glucose disposal. Subcellular fractionation of skeletal muscles suggested that these reductions result from impaired translocation of Glut 4 to the plasma membrane, providing powerful evidence that syntaxin 4 is the t-SNARE required for Glut4 vesicle docking at the skeletal muscle plasma membrane. Surprisingly, however, insulin-stimulated Glut4 translocation is not impaired in adipocytes isolated from Syn 4 heterozygous knockout mice. The authors suggest that these observations can be reconciled if adipocytes express a functional surplus of syntaxin 4, whereas skeletal muscles express a limiting quantity of the t-SNARE.

\section{New protein targets for the enhancement of insulin sensitivity?}

Insulin resistance is an extremely prevalent condition in many human populations, being closely associated with obesity and NIDDM. Because the molecular basis of insulin resistance is likely to be heterogeneous, any new animal model of insulin resistance can be of considerable experimental value. If human muscle tissue resembles that of mice with respect to the expression of syntaxin 4 and its functional limitation on Glut 4 translocation, any genetic condition that downregulates the activity or level of syntaxin 4 is likely to predispose to insulin resistance. It will be of interest to determine whether other components implicated in Glut 4 trafficking in the adipocyte also play a role in skeletal muscle, and to ascertain whether any of these factors are also expressed in limiting quantities in muscle. In this regard, Khan et al. (29) have recently shown that overexpression of Munc18c inhibits insulin-stimulated Glut4 translocation to t-tubules but not to the sarcolemma in skeletal muscle. This finding is consistent with the observation that $\mathrm{t}$ tubules, as compared with the sarcolemma, contain a lesser quantity of syntaxin 4. Presumably, the overexpression of Munc18c was sufficient to saturate the syntaxin 4 present in t-tubules but not to significantly inhibit Glut4 vesicle docking at the sarcolemma because of the higher concentration of the t-SNARE in this membrane.
Even under maximally stimulating conditions, a sizeable fraction of Glut4 appears to remain in intracellular compartments. Therefore, an interesting implication of the present results (4) is that overexpression of syntaxin 4 may augment the translocation of Glut4 vesicles to the plasma membrane in skeletal muscle and thus enhance insulin sensitivity. If so, syntaxin 4 expression may represent a new target for therapies designed to counteract insulin resistance and diabetic hyperglycemia. Upregulation of limiting components involved in Glut4 translocation may be especially effective when combined with the therapeutic upregulation of Glut4, which, in mouse models, can prevent the development of insulin resistance and NIDDM (30).

\section{Glut4 trafficking in skeletal muscle and adipocytes: identical mechanisms?}

The data of Yang et al. (4) and of Khan et al. (29) demonstrate, unsurprisingly, perhaps, that some of the proteins involved in Glut 4 trafficking are required in both muscle fibers and adipocytes. A more fundamental question is whether the mechanisms in the two cell types are identical. This would appear to be unlikely, both because of the greater complexity of the muscle fiber, with its additional membrane domain, and because the intracellular reservoir from which Glut4 translocates to the plasma membrane appears to differ between these cell types. In both adipocytes (14) and muscle fibers (28), elements of the trans-Golgi network appear to store Glut4 prior to its appearance on the cell surface. However, it is unclear in either case whether the trans-Golgi contains the immediate precursor pool for translocating Glut4, or whether it represents a replenishing reservoir for the immediate precursor pool. It is also unclear whether a single immediate precursor pool exists in either adipocytes or muscle fibers, or whether translocation to t-tubules or the sarcolemma occurs from the same precursor pool or from two different pools.

Given the subcellular architecture of muscle fibers, the trans-Golgi is not a likely immediate reservoir for the translocation of Glut4 to t-tubules. Nearly all of the Golgi structures discernible in muscle fibers lie in close proximity to nuclei just beneath the sarcolemma, whereas t-tubules can extend 
great distances into the muscle fiber, separated from the bulk of trans-Golgi structures by imposing physical barriers consisting of the myofibrillar network and the membranes of the sarcoplasmic reticulum network. Wang et al. (27) identified a substantial pool of Glut 4 in the terminal cisternae that surround the t-tubules. Structurally, this would appear to represent a good immediate pool for the translocation of Glut4 to ttubules, but there is no direct evidence supporting this suggestion. Finally, signals appear to be recognized differently in cultured myocytes (31) and in adipocytes (32). Resolving this question will require a more thorough characterization of insulin-dependent vesicle trafficking, especially in the muscle fiber.

\section{The dominant role of $t$-tubules in insulin-stimulated glucose uptake}

The observations of Yang et al. (4) and of Khan et al. (29) support previous findings that t-tubules play a dominant role in insulin-stimulated glucose transport in skeletal muscle (27). Downregulation of syntaxin 4 by $50 \%$ should decrease Glut4 translocation to and glucose transport across t-tubules in skeletal muscle but should not affect these parameters at the sarcolemma, which contains an excess of the tSNARE (29). The results of Yang et al. (4) demonstrate that a decrease in syntaxin 4 protein in t-tubules is associated with a proportional decrease in both insulin-stimulated muscle glucose uptake and whole-body glucose disposal in heterozygous Syn 4 null mice. This close correlation argues that the bulk of insulin-stimulated glucose uptake occurs across t-tubules and, further, that uptake across the sarcolemma during insulin stimulation is relatively low.

1. Mueckler, M. 1994. Facilitative glucose transporters. Eur. J. Biochem. 219:713-725.

2. Cline, G.W., et al. 1999. Impaired glucose transport as a cause of decreased insulin-stimulated muscle glycogen synthesis in type 2 diabetes. $N$. Engl. J. Med. 341:240-246.
3. Warram, J.H., Martin, B.C., Krolweski, A.S., Soeldner, J.S., and Kahn, C.R. 1990. Slow glucose removal rate and hyperinsulinemia precede the development of type II diabetes in the offspring of diabetic parents. Ann. Intern. Med. 113:909-915.

4. Yang, C., et al. 2001. Syntaxin 4 heterozygous knockout mice develop muscle insulin resistance. J. Clin. Invest. 107:xxx-yyy

5. Cushman, S.W., and Wardzala, L.J. 1980. Potential mechanism of insulin action on glucose transport in the isolated rat adipose cell. Apparent translocation of intracellular transport systems to the plasma membrane. J. Biol. Chem. 255:4758-4762.

6. Suzuki, I., and Kono, T. 1980. Evidence that insulin causes translocation of glucose transport activity to the plasma membrane from an intracellular storage site. Proc. Natl. Acad. Sci. USA. 77:2542-2545.

7. James, D.E., Strube, M., and Mueckler, M. 1989 Molecular cloning and characterization of an insulin regulatable glucose transporter. Nature. 338:83-87.

8. Birnbaum, M.J. 1989. Identification of a novel gene encoding an insulin-responsive glucose transporter protein. Cell. 57:305-315.

9. Charron, M.J., Brosius, F.D., Alper, S.L., and Lodish, H.F. 1989. A glucose transport protein expressed predominately in insulin-responsive tissues. Proc. Natl. Acad. Sci. USA. 86:2535-2539.

10. Zorzano, A., et al. 1989. Insulin-regulated glucose uptake in rat adipocytes is mediated by two transporter isoforms present in at least two vesicle populations. J. Biol. Chem. 264:12358-12363.

11. Holman, G.D., et al. 1990. Cell surface labeling of glucose transporter isoform GLUT4 by bis-mannose photolabel. Correlation with stimulation of glucose transport in rat adipose cells by insulin and phorbol ester. J. Biol. Chem. 265:18172-18179.

12. Jhun, B.H., Rampal, A.L., Liu, H., Lachaal, M., and Jung, C.Y. 1992. Effects of insulin on steady state kinetics of GLUT4 subcellular distribution in rat adipocytes. Evidence of constitutive GLUT4 recycling. J. Biol. Chem. 267:17710-17715.

13. Czech, M.P., and Buxton, J.M. 1993. Insulin action on the internalization of the GLUT4 glucose transporter in isolated rat adipocytes. J. Biol. Chem. 268:9187-9190.

14. Slot, J.W., Geuze, H.J., Gigengack, S., Lienhard, G.E., and James, D.E. 1991. Immuno-localization of the insulin regulatable glucose transporter in brown adipose tissue of the rat. J. Cell Biol. 113:123-135.

15. Slot, J.W., Geuze, H.J., Gigengack, S., James, D.E., and Lienhard, G.E. 1991. Translocation of the glucose transporter GLUT4 in cardiac myocytes of the rat. Proc. Natl. Acad. Sci. USA. 88:7815-7819.

16. Pessin, J.E., Thurmond, D.C., Elmendorf, J.S., Coker, K.J., and Okada, S. 1999. Molecular basis of insulin-stimulated GLUT4 vesicle trafficking. Location! Location! Location! J. Biol. Chem. 274:2593-2596.

17. Martin, L.B., Shewan, A., Millar, C.A., Gould, G.W., and James, D.E. 1998. Vesicle-associated membrane protein 2 plays a specific role in the insulin-dependent trafficking of the facilitative glucose transporter GLUT4 in 3T3-L1 adipocytes. J. Biol. Chem. 273:1444-1452.

18. Olson, A.L., Knight, J.B., and Pessin, J.E. 1997. Syntaxin 4, VAMP2, and/or VAMP3/cellubrevin are functional target membrane and vesicle SNAP receptors for insulin-stimulated GLUT4 translocation in adipocytes. Mol. Cell. Biol. 17:2425-2435

19. Rea, S., et al. 1998. Syndet, an adipocyte target SNARE involved in the insulin-induced translocation of GLUT4 to the cell surface. J. Biol. Chem. 273:18784-18792.

20. Tamori, Y., et al. 1998. Inhibition of insulininduced GLUT4 translocation by Munc18c through interaction with syntaxin 4 in 3T3-L1 adipocytes. J. Biol. Chem. 273:19740-19746.

21. Thurmond, D.C., et al. 1998. Regulation of insulinstimulated GLUT4 translocation by Munc18c in 3T3L1 adipocytes. J. Biol. Chem. 273:33876-33883.

22. Min, J., et al. 1999. Synip: a novel insulin-regulated syntaxin 4-binding protein mediating GLUT4 translocation in adipocytes [erratum 1999, 4:192]. Mol. Cell. 3:751-760.

23. Yeh, J.I., Verhey, K.J., and Birnbaum, M.J. 1995. Kinetic analysis of glucose transporter trafficking in fibroblasts and adipocytes. Biochemistry. 34:15523-15531.

24. Marette, A., et al. 1992. Abundance, localization, and insulin-induced translocation of glucose transporters in red and white muscle. Am. J. Physiol. 263:C443-C452.

25. Hansen, P.A., et al. 1995. Skeletal muscle glucose transport and metabolism are enhanced in transgenic mice overexpressing the Glut4 glucose transporter. J. Biol. Chem. 270:1679-1684.

26. Rahkila, P., Luukela, V., Vaananen, K., and Metsikko, K. 1998. Differential targeting of vesicular stomatitis virus $G$ protein and influenza virus hemagglutinin appears during myogenesis of L6 muscle cells. J. Cell Biol. 140:1101-1111.

27. Wang, W., Hansen, P.A., Marshall, B.A., Holloszy, J.O., and Mueckler, M. 1996. Insulin unmasks a $\mathrm{COOH}$-terminal Glut4 epitope and increases glucose transport across T-tubules in skeletal muscle. J. Cell Biol. 135:415-430.

28. Ploug, T., van Deurs, B., Ai, H., Cushman, S.W., and Ralston, E. 1998. Analysis of GLUT4 distribution in whole skeletal muscle fibers: identification of distinct storage compartments that are recruited by insulin and muscle contractions. $J$. Cell Biol. 142:1429-1446.

29. Khan, A.H., et al. 2001. Munc18c regulates insulin-stimulated GLUT4 translocation to the transverse tubules in skeletal muscle. J. Biol. Chem. 276:4063-4069.

30. Marshall, B.A., Hansen, P.A., Ensor, N.J., Ogden, M.A., and Mueckler, M. 1999. GLUT-1 or GLUT4 transgenes in obese mice improve glucose tolerance but do not prevent insulin resistance. $A m$ J. Physiol. 276:E390-E400.

31. Haney, P.M., Levy, M.A., Strube, M.S., and Mueckler, M. 1995. Insulin-sensitive targeting of the GLUT4 glucose transporter in L6 myoblasts is conferred by its $\mathrm{COOH}$-terminal cytoplasmic tail J. Cell Biol. 129:641-658.

32. Marsh, B.J., Alm, R.A., McIntosh, S.R., and James, D.E. 1995. Molecular regulation of GLUT-4 targeting in 3T3-L1 adipocytes. J. Cell Biol. 130:1081-1091. 